\title{
Pacific
}

Journal of

Mathematics

\section{ON THE FAILURE CYCLES FOR THE QUADRATIC NORMALITY OF A PROJECTIVE VARIETY}

EDOARDO BALLICO 


\title{
ON THE FAILURE CYCLES FOR THE QUADRATIC NORMALITY OF A PROJECTIVE VARIETY
}

\author{
EDOARDO BALLICO
}

Let $X$ be a smooth projective surface and $L$ a very ample line bundle on $X$ which is not quadratically normal; set $r+1=h^{0}(X, L)$. Here we give numerical conditions on $X$ and $L$ which imply the existence of a finite subscheme $T$ of $X$ with length $(T) \geq 2 s+2$ and contained in a dimension $s \leq r-2$ linear subspace of $P\left(H^{0}(X, L)\right)$ and such that $L \mid T$ is not quadratically normal.

\section{Introduction.}

It is very classical the following problem (with several variations). Suppose that a curve $C \subset \mathbf{P}^{r}$ has some bad property, e.g. it is not projectively normal. Show the existence of a finite subscheme $S$ of $C$ contained in a smaller linear subspace such that $S$ explains the failure of $C$ to be projectively norrnal. In modern times there is the important paper [4]. Here we consider the corresponding problem when the scheme $C$ has $\operatorname{dim}(C)>1$. We were also motivated from the notion of $\mathrm{k}$-ampleness and $\mathrm{k}$-very ampleness introduced in [2]. By definition these conditions fail for a scheme $C$ if and only if there is a zero dimensional subscheme $S$ of $C$ with a bad property. We were interested (see e.g. [1]) in showing that under suitable conditions there are many such subschemes. A natural question was if there is some bad positive dimensional proper subscheme $Y$ containing all of them for a natural reason (for example if it were the union of them) or if there was some bad "free" zero dimensional subscheme. Here we consider the condition of quadratic normality and give a positive answer if $\operatorname{dim}(C)=2$ under suitable numerical conditions. These numerical conditions are strange, far from optimal and just come from the proof. We will state them below as Theorem 0.2. But first and most important: the proofs are essentially technical variations on an alternative proof $([5, \S 2.5])$ of a theorem in [4]; hence the idea originates ultimately with Robert Lazarsfeld. After the present results were proven, we checked the references and found that exactly that subsection was deleted in the printed version [6] of [5]. After a while we decided to rewrite a little bit the paper, but to write it anyway. 
We fix an integral variety $X$ and a very ample line bundle $L$ on $X$; set $r+1:=h^{0}(X, L)$ and $\mathbf{O}:=\mathbf{O}_{X}$; let $\phi_{L}: X \rightarrow \mathbf{P}^{r}$ be the embedding associated to $H^{0}(X, L)$ into a projective space. Recall that a subvariety $U$ of $\mathbf{P}(V)$ is called quadratically normal if the restriction map $V \otimes V \rightarrow$ $H_{0}\left(U, \mathbf{O}_{U}(2)\right)$ is surjective. The pair $(X, L)$ (or just $\left.L\right)$ is called quadratically normal if $\phi_{L}(X)$ is quadratically normal.

Definition 0.1. If $L$ is not quadratically normal, we will call amount of failure of quadratic normality the integer $\operatorname{dim}\left(\operatorname{coker}\left(H^{0}(L) \otimes H^{0}(L) \rightarrow\right.\right.$ $\left.H^{0}\left(L^{2}\right)\right)$.

Let $G=G(r+1-\operatorname{dim}(X), r+1)$ be the Grassmannian of codimension $\operatorname{dim}(X)$ linear subspaces of $\mathbf{P}^{r}$; set

$$
B:=\{U \in G: X \cap U \text { is not zero dimensional }\} .
$$

Here is the main result proven in this paper.

Theorem 0.2. Assume $\operatorname{dim}(X)=2$ and that $L$ is not quadratically normal. Let $\mathbf{f}>0$ be the amount of failure of the quadratic normality of $X$. If $h^{1}\left(\mathbf{O}_{X}\right)<\mathbf{f}+\operatorname{codim}(B)-1$, then there is a codimension 2 linear subspace $[U] \in G \backslash B$ such that the scheme $X \cap U$ is 0-dimensional and is not quadratically normal with respect to $L \mid(X \cap U)$. Furthermore, there is a an integer $s \leq r-2$, a linear subspace $V$ of $U$ with $\operatorname{dim}(V)=s$ and a subscheme $T$ of $U \cap X$ contained in $V$ with length $(T)=2 s+2$ such that $T$ is not quadratically normal with respect to $L \mid T$.

In particular Theorem 0.2 applies to all linearly normal but not quadratically normal embedded surfaces with $h^{1}\left(\mathbf{O}_{X}\right)=0$.

For other related 1 results proven within the same framework, see 2.2 and 2.3. In $\S 1$ (after fixing the notations) we will give the framework and the main ingredients for the proofs of all the results of this paper. In $\S 2$ we will prove Theorem 0.2 .

The author owes a huge debt to the referee for essential constructive criticism and for fundamental mathematical contributions which improved the original statement of 0.2 .

The author was partially supported by MURST and GNSAGA of CNR (Italy).

\section{Preliminaries and general set up.}

We work over an algebraically closed base field. We fix an integral variety $X$ and a very ample line bundle $L$ on $X$; set $r+1:=h^{0}(X, L)$; let $\phi_{L}: X \rightarrow \mathbf{P}^{r}$ the embedding associated to $H^{0}(X, L)$. If $A$ is a sheaf on $X$, we will often 
write $H^{i}(A)$ or $h^{i}(A)$ for $H^{i}(X, A)$ or $h^{i}(A)$. Set $Y:=\phi_{L}(X)$. Let $\Omega$ be the cotangent sheaf of $\mathbf{P}^{r}$. Set $M_{L}:=\phi_{L}{ }^{*}(\Omega(1))$. By the dual of the Euler sequence of $T \mathbf{P}^{r}$ and the completeness of the embedding of $X$ we obtain the following exact sequence on $X$ :

$$
0 \rightarrow M_{L} \rightarrow H^{0}(X, L) \otimes \mathbf{O}_{X} \rightarrow L \rightarrow 0
$$

which contains a lot of informations on the cohomology of $I_{Y}$.

Now we generalize the Remark in [6] given at page 510 (between the statement of [6], Prop. 1.3.3, and its proof).

Lemma 1.1. With the notations $X, L, \phi_{L}, M_{L}$, and so on introduced at the beginning, we have:

(i) Fix an integer $k>0$ and assume $H^{1}\left(L^{k}\right)=0$; the multiplication map $H^{0}(L) \otimes H^{0}\left(L^{k}\right) \rightarrow H^{0}\left(L^{k+1}\right)$ is surjective if and only if $H^{1}\left(M_{L} \otimes L^{k}\right)=$ 0 . In particular if $h^{1}\left(L^{s}\right)=0$ for every $s>0$, then $L$ is normally generated if and only if $H^{1}\left(M_{L} \otimes L^{t}\right)=0$ for every $t>0$.

(ii) The amount of failure for the quadratic normality of $L$ is

$$
\operatorname{dim}\left(\operatorname{ker}\left(H^{1}\left(M_{L} \otimes L\right) \rightarrow H^{0}(L) \otimes H^{1}(L)\right)\right) .
$$

(iii) If $H^{1}\left(L^{2}\right)=0$ the amount of failure of quadratic normality is

$$
h^{1}\left(M_{L} \otimes L\right)-h^{0}(L) \cdot h^{1}(L) .
$$

Proof. Just use a twist of the exact sequence (1).

Let $G:=G(r-x+1, r+1)$ be the Grassmannian of codimension $x$ linear subspaces of $\mathbf{P}\left(H^{0}(X, L)\right)$ and $F:=\{(y, U) \in X \times G: y \in U\} \subset X \times G$ be the incidence variety. On $G$ we have the exact sequence

$$
0 \rightarrow S \rightarrow H^{0}(X, L) \otimes \mathbf{O}_{G} \rightarrow Q \rightarrow 0
$$

with $Q$ tautological quotient bundle and $S$ tautological rank $x$ subbundle. Let $f: X \times G \rightarrow G$ and $p: X \times G \rightarrow X$ be the projections. The incidence variety $F$ is defined by the vanishing of the induced morphism $s: f^{*} S \rightarrow p^{*} L$ i.e., its ideal sheaf $\mathbf{I}$ in $X \times G$ is the image of the associated map $f^{*} S \otimes p^{*} L^{*} \rightarrow \mathbf{O}_{X \times G}$. Note that this ideal sheaf $\mathbf{I}$ has a resolution:

$$
\begin{gathered}
\cdots f^{*} \Lambda^{t+1} S \otimes p^{*} L^{* t} \rightarrow f^{*} \Lambda^{t} S \otimes p^{*} L^{*(t-1)} \rightarrow f^{*} \Lambda^{(t-1)} S \otimes p^{*} L^{(t-2)} \ldots \\
\cdots \rightarrow f^{*} \Lambda^{2} S \otimes p^{*} L^{*} \rightarrow f^{*} S \rightarrow \mathbf{I} \otimes p^{*} L \rightarrow 0 .
\end{gathered}
$$

On $X \times G$ there is an important commutative diagram. First, we will write it as formula (4) in the particular case $x=\operatorname{rank}(S)=2$ needed in the proof 
of 0.2 .

(4)

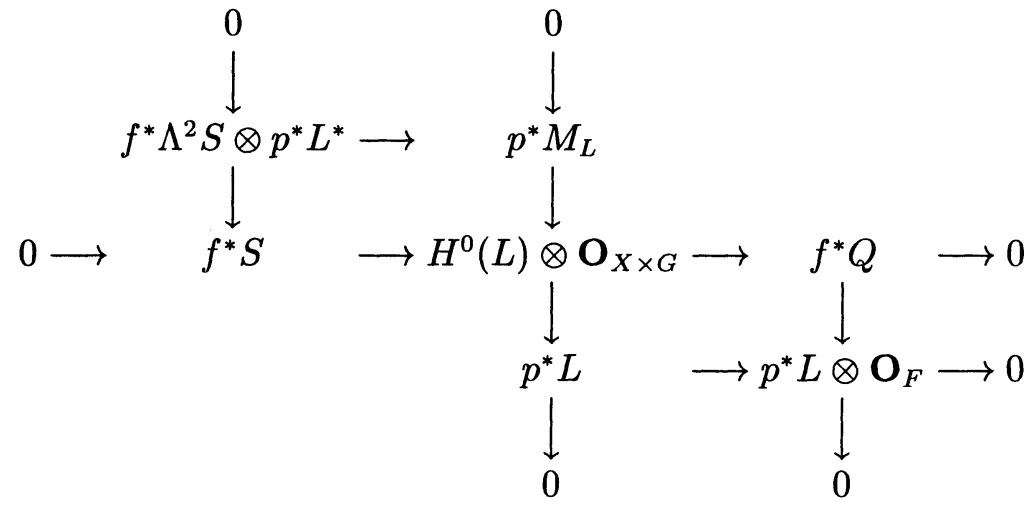

In the general case this commutative diagram has 3 columns. The first column of this diagram is the resolution (3) of $\mathbf{I}$ (without $\mathbf{I}$ ). The second column of the diagram is the pull-back $p^{*}$ of the exact sequence (1) and the third column is just the tautological surjection $f^{*} Q \rightarrow p^{*} L \otimes \mathbf{O}_{F}$. These columns are connected so that the only long row in the diagram is the pullback by $f^{*}$ of the exact sequence (1); just above this exact sequence there is a map $f^{*} \Lambda^{2} S \otimes p^{*} L^{*} \rightarrow p^{*} M_{L}$ and just below the exact sequence there is the surjection $p^{*} L \rightarrow p^{*} L \otimes \mathbf{O}_{F}$ coming from the surjection $\mathbf{O}_{X \times G} \rightarrow \mathbf{O}_{F}$. Follow the first column of the diagram till the term $f^{*} \Lambda^{2} S \otimes p^{*} L^{*}$; then go on the right one step and find $f^{*} Q$; then go down one step and find $f^{*} \Lambda^{2} S \otimes p^{*} L^{*}$. In this way from this diagram we obtain an exact sequence obtained from the exact sequence (3) substituting the last part $f^{*} S \rightarrow \mathbf{I} \otimes p^{*} L \rightarrow 0$ with

$$
p^{*} M_{L} \rightarrow f^{*} Q \rightarrow p^{*} L \otimes \mathbf{O}_{F} \rightarrow 0 .
$$

Call $(\S \S)(\mathrm{k})$ the exact sequence obtained twisting by $p^{*} L_{k}$ the sequence just described. If $x=2$ the complex $(\S \S)(1)$ is the following exact sequence:

$$
0 \rightarrow f^{*} \Lambda^{2} S \rightarrow p^{*}\left(M_{L} \otimes L\right) \rightarrow f^{*} Q \otimes p^{*} L \rightarrow p^{*} L^{2} \otimes \mathbf{O}_{F} \rightarrow 0 .
$$

Now we push-forward the complex $(\S \S)(1)$ to the Grassmannian; since $(\S \S)(1)$ is exact, its higher pushforwards vanish and we obtain a spectral sequence (call it (\#)) converging to zero.

$$
E_{1}^{q t}=R^{t} f_{*}\left(C^{q}\right) \Rightarrow 0
$$

where

$$
0 \rightarrow C^{2-x} \rightarrow \cdots \rightarrow C^{0} \rightarrow C^{1} \rightarrow C^{2} \rightarrow C^{3} \rightarrow 0
$$


is the complex

$0 \rightarrow f^{*} \Lambda^{x} S \otimes p^{*} L^{*(x-2)} \rightarrow \cdots \rightarrow f^{*} \Lambda^{2} S \rightarrow p^{*}\left(M_{L} \otimes L\right) \rightarrow f^{*} Q \otimes p^{*} L \rightarrow p^{*} L^{2} \otimes \mathbf{O}_{F} \rightarrow 0$.

In Section 2 we will write the $E_{1}$-part of (\#) as formulas (7), (8) and (9) in the case $\operatorname{dim}(X)=x=\operatorname{rank}(S)=2$ we need for the proof of 0.2 . Use the projection formula $R^{i} f_{*}\left(f^{*} A^{\prime} \otimes p^{*} A\right)=H^{i}(X, A) \otimes A^{\prime}$ for all locally free sheaves $A$ on $X$. We normalize the indices of the complex $(\S \S)(1)$ in such a way that the term $E_{1}^{00}$ of the spectral sequence (\#) is $H^{0}\left(X, \mathbf{O}_{X}\right) \otimes \Lambda^{2} S$. With this normalization the term $E_{1}^{q t}$ of (\#) is 0 if either $t<0$ or $q<2-x$, it is $H^{t}\left(X, \mathbf{O}_{X}\right) \otimes \Lambda^{-q+2} S$ for $2-x \leq q \leq 0, H^{t}\left(X, M_{L} \otimes L\right) \otimes \mathbf{O}_{G}$ for $q=1, H^{t}(X, L) \otimes Q$ for $q=2$ and $R^{t} f_{*}\left(p^{*} L^{2} \otimes \mathbf{O}_{F}\right)$ for $q=3$.

Remark 1.2. Note that over $G \backslash B$ we have $R^{i} f_{*}\left(p^{*} L^{j} \otimes \mathbf{O}_{F}\right)=0$ for every $j$ and every $i>x-2$ because the fibers of $f$ over $G \backslash B$ have dimension $\leq x-2$. Fix a point $[U] \in G \backslash B$ corresponding to a codimension $x$ linear subspace $U$ of $\mathbf{P}^{r}$. Then for every integer $k$ the fiber of the sheaf $f_{*}\left(p^{*} L^{k+1} \otimes \mathbf{O}_{F}\right)$ at $[U]$ is canonically isomorphic to the vector space $H^{0}\left(U, \mathbf{O}_{U \cap X}(k+1)\right)$ and fiber over $[U]$ of the homomorphism $u:=d_{1}^{20}: H^{0}(X, L) \otimes Q \rightarrow f_{*}\left(p^{*} L^{2} \otimes \mathbf{O}_{F}\right)$ in the $E_{1}$-part of the spectral sequence (\#) is identified at $[U]$ with the natural multiplication map

$$
H^{0}(X, L) \otimes H^{0}\left(U, \mathbf{O}_{U}(1)\right) \rightarrow H^{0}\left(U \mathbf{O}_{X \cap U}(2)\right) .
$$

\section{Proof of Theorem 0.2.}

Now we specialize the situation of $\S 1$ to the situation of Theorem 0.2 , whose proof will be given now.

Proof of Theorem 0.2. First, note that the "Furthermore part" of the statement of 0.2 follows from the first part and [6, Lemma 2.4.4].

Now we will prove the first part of 0.2 . We write as formulas (7), (8) and (9) the 3 non trivial lines of the $E_{1}$-term of the spectral sequence (\#) under the assumptions of 0.2 ; in particular we have $x=2, \operatorname{rank}(S)=2, \Lambda^{2} S \cong$ $\mathbf{O}_{G}(-1), \operatorname{dim}(X)=2$.

$$
H^{2}\left(\mathbf{O}_{X}\right) \otimes \mathbf{O}_{G}(-1) \rightarrow H^{2}\left(M_{L} \otimes L\right) \otimes \mathbf{O}_{G} \rightarrow H^{2}(L) \otimes Q
$$

$H^{1}\left(\mathbf{O}_{X}\right) \otimes \mathbf{O}_{G}(-1) \stackrel{\alpha}{\rightarrow} H^{1}\left(M_{L} \otimes L\right) \otimes \mathbf{O}_{G} \stackrel{\beta}{\rightarrow} H^{1}(L) \otimes Q \rightarrow R^{1} f_{*}\left(p^{*} L^{2} \otimes \mathbf{O}_{F}\right)$

(9) $H^{0}\left(\mathbf{O}_{X}\right) \otimes \mathbf{O}_{G}(-1) \rightarrow H^{0}\left(M_{L} \otimes L\right) \otimes \mathbf{O}_{G} \rightarrow H^{0}(L) \otimes Q \stackrel{u}{\rightarrow} R^{0} f_{*}\left(p^{*} L^{2} \otimes \mathbf{O}_{F}\right)$. 
Let $\alpha:=d_{1}^{01}, \beta:=d_{1}^{11}, u:=d_{1}^{20}$ be the maps indicated above. By Remark 1.2 to prove 0.2 it is sufficient to prove that the map $u$ is not surjective on $G \backslash B$. We use that the spectral sequence (\#) converges to 0 because the complex $(\S \S)(1)$ is exact. We have $\operatorname{coker}(u)=E_{2}^{30}$. We divide the proof into two parts.

(A) Here we assume $h^{1}(L)=0$, hence $\beta=0$ and $\operatorname{coker}(a)=E_{2}^{11}$. Since the spectral sequence (\#) abuts to 0 , we have

$$
0=E_{\infty}^{11}=E_{3}^{11}=\operatorname{ker}\left(d_{2}^{11}: E_{2}^{11} \rightarrow E_{2}^{30}\right) .
$$

Hence $\operatorname{coker}(\alpha)$ injects onto $\operatorname{coker}(u)$. Hence it is sufficient to prove that the codimension of the support of $\operatorname{coker}(\alpha)$ is at most $h^{1}\left(\mathbf{O}_{X}\right)-\mathbf{f}+1$. Since $\alpha$ : $H^{1}\left(\mathbf{O}_{X}\right) \otimes \mathbf{O}_{G}(-1) \rightarrow \mathbf{O}_{G}^{\mathbf{f}}$ and $\mathbf{O}_{G}(1)$ is ample, this follows from [3, Th. 1.l(a)].

(B) Now we make no assumption on $H^{1}(L)$. As in the corresponding case of [5], the exact sequence (1) gives a homomorphism

$$
c: H^{1}\left(M_{L} \otimes L\right) \rightarrow H^{1}(L) \otimes H^{0}(L)
$$

and $\operatorname{dim}(\operatorname{ker}(c))$ is the amount of failure $\mathbf{f}$ of quadratic normality of $L$ by Lemma 1.1 (ii). On $G$ there is an inclusion of sheaves $(\operatorname{ker}(c)) \otimes \mathbf{O}_{G} \rightarrow \operatorname{ker}(\beta)$. Since $\operatorname{ker}(\beta)$ is a subsheaf of a trivial sheaf, this inclusion is an isomorphism of $(\operatorname{ker}(c)) \otimes \mathbf{O}_{G}$ onto a direct summand of $\operatorname{ker}(\beta)$. Hence projecting $\operatorname{ker}(\beta)$ onto this summand we obtain a surjection from $E_{2}^{11}=\operatorname{ker}(\beta) / \operatorname{im}(\alpha)$ onto $\operatorname{coker}\left(H^{1}\left(\mathbf{O}_{X}\right) \otimes \mathbf{O}_{G}(-1) \rightarrow \mathbf{O}_{G}^{\mathbf{f}}\right)$. We conclude as in part (A).

The proof of 0.2 is over.

Remark 2.1. The proof of 0.2 depends only on $\operatorname{dim}(B)$. If we want to exclude a bigger subset of $G$, then we obtain a corresponding result in a suitable range. Viceversa, if we may control a dense part of $B$ the corresponding result is true in a larger range. The proof of Theorem 0.2 gives with no change the following result.

Proposition 2.2. Fix an integer $k \geq 1$. Assume $\operatorname{dim}(X)=2$. Assume the surjectivity of the restriction map $H^{0}\left(\mathbf{P}^{r}, \mathbf{O}_{\mathbf{P}}(k)\right) \rightarrow H^{0}\left(\phi_{L}(X), \mathbf{O}(k)\right) \cong$ $H^{0}\left(L^{k}\right)$. Let $\mathbf{f}(k)$ be the dimension of the cokernel of the multiplication map $H^{0}(L) \otimes H^{0}\left(L^{k}\right) \rightarrow H^{0}\left(L^{k+1}\right)$. Assume $\mathbf{f}(k)>0$ and

$$
h^{1}\left(L^{k-1}\right)<\mathbf{f}(k)+\operatorname{codim}(B)-1 .
$$

Then there is a codimension 2 linear subspace $[U] \in G \backslash B$ such that the scheme $X \cap U$ is 0-dimensional and the multiplication map

$$
H^{0}(L) \otimes H^{0}\left(X \cap U,(L \mid(X \cap U))^{k}\right) \rightarrow H^{0}\left(X \cap U,(L \mid(X \cap U))^{k+1}\right)
$$


is not surjective.

Remark 2.3. $\quad$ Note that for a complete but not projectively normal embedding the machine can start (and give informations on $(X, L))$ using the proposition just given exactly at the first step, say the $(k+1)^{\text {th }}$ step, at which the embedding is not $(k+1)$-normal. However, it can also be used at an intermediate step with large $h^{0}\left(L^{k}\right)$, obtaining a result of Castelnuovo Mumford type.

If we look at the proof of Theorem 0.2 when $X$ is a smooth curve with $H^{1}(L) \leq 1$, we find exactly the proof of $[5, \S 2.5]$. In the statement we have the small precision about the amount of failure of quadratic normality of $L$.

\section{References}

[1] E. Ballico, On the failure locus of higher order properties of embeddings in projective spaces, Math. Nachr., 163 (1993), 5-13.

[2] M. Beltrametti, P. Francia and A. J. Sommese, On Reider's method and higher order embeddings, Duke Math. J., 58 (1989), 425-439.

[3] W. Fulton and R. Lazarsfeld, On the connectedness of degeneracy loci and special divisors, Acta Math., 146 (1981), 271-283.

[4] M. Green and R. Lazarsfeld, On the projective normality of complete linear series on an algebraic curve, Invent. Math., 83 (1986), 73-90.

[5] R. Lazarsfeld, A sampling of vector bundle techniques in the study of linear series, preliminary version of [6] distributed to the participants of the "College on Riemann Surfaces", ICTP, Trieste, Italy, 9 Nov.-18 Dec. 1987.

[6] — A sampling of vector bundle techniques in the study of linear series, in: Lectures on Riemann Surfaces, Proc. College on Riemann Surfaces, ICTP (Trieste, Italy, 9 Nov.-18 Dec. 1987, pp. 500-559), World Scientific, 1989.

Received July 14, 1993 and revised November 18, 1994.

UNIVERSITY OF TRENTO

ITALY 

Rosa M. Miró-Roig, Singular moduli spaces of stable vector bundles on $\mathbf{P}^{3}$

Hitoshi Moriyoshi and Toshikazu Natsume, The Godbillon-Vey Cyclic Cocycle and Longitudinal Dirac Operators

J.C. Naranjo, The positive dimensional fibres of the Prym map

Artur Nicolau and Arne Stray, Nevanlinna's coefficients and Douglas al-

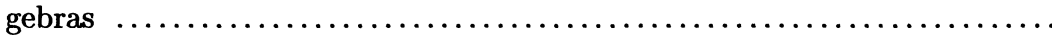

K.K. Park, Entropy of a skew product with a $Z^{2}$-action $\ldots \ldots \ldots \ldots \ldots .227$

María Cristina Pereyra, Sobolev spaces on Lipschitz curves ......... 553

T. Sano, Commuting co-commuting squares and finite dimensional Kac algebras

H.B. Thompson, Second order ordinary differential equations with fully nonlinear two point boundary conditions

H.B. Thompson, Second order ordinary differential equations with fully nonlinear two point boundary conditions II

F. $\mathrm{Xu}$, The flat part of non-flat orbifolds

Hidenobu Yoshida, A type of uniqueness for the Dirichlet problem on a half-space with continuous data 


\section{PACIFIC JOURNAL OF MATHEMATICS}

Volume $172 \quad$ No. $2 \quad$ February 1996

On the failure cycles for the quadratic normality of a projective variety

307

EdoARdo BaLlico

On the minimal free resolution of general embeddings of curves

315

EdOARDO BALlico

On normality of the closure of a generic torus orbit in $G / P$

321

ROMUALD DABROWSKI

Paragroupe d'Adrian Ocneanu et algèbre de Kac

331

MARIE-Claude DAVID

Irreducibility and dimension theorems for families of height 3 Gorenstein algebras

SuSAN J. DiESEL

On the cohomology of the Lie algebra $L_{2}$

ALICE FIALOWSKI

Generic differentiability of convex functions on the dual of a Banach space

John R. Giles, P. S. Kenderov, WarRen Brian Moors and S. D.

SCIFFER

Moon hypersurfaces and some related existence results of capillary hypersurfaces

without gravity and of rotational symmetry

FEI-TSEN LIANG

Stable relations. II. Corona semiprojectivity and dimension-drop $C^{*}$-algebras

TERRY ATHERTON LORING

Singular moduli spaces of stable vector bundles on $\mathbf{P}^{3}$

Rosa M. MIRó-ROIG

The Godbillon-Vey cyclic cocycle and longitudinal Dirac operators

Hitoshi MoRIYOSHI and TOSHIKAZU NATSUME

Nevanlinna's coefficients and Douglas algebras

ARTUR NiCOLAU and ARNE STRAY

Sobolev spaces on Lipschitz curves

MARÍA CRISTINA PEREYRA

A type of uniqueness for the Dirichlet problem on a half-space with continuous data 